\title{
Detection of biocolloids in aquatic media by Nano-Particle Analyzer
}

\author{
T. Bundschuh ${ }^{\text {a,* }}$, T. Wagner ${ }^{\mathrm{a}}$, I. Eberhagen ${ }^{\mathrm{b}}$, B. Hambsch ${ }^{\mathrm{b}}$ and R. Köster ${ }^{\mathrm{a}}$ \\ ${ }^{a}$ Forschungszentrum Karlsruhe GmbH, Institute for Technical Chemistry, Water Technology and \\ Geotechnology Division, PO Box 3640, D-76021 Karlsruhe, Germany \\ ${ }^{\mathrm{b}}$ Technologiezentrum Wasser, Division of Microbiology, Karlsruher Straße 84, D-76139 Karlsruhe, \\ Germany
}

\begin{abstract}
The Nano-Particle Analyzer (NPA) based on Laser-Induced Breakdown Detection (LIBD) selectively generates and detects plasma events on colloids in aquatic media. Here, it is made use of the fact that the power density required for plasma generation decreases from the gaseous to the solid medium. At an adequate laser pulse energy, plasmas can thus be generated selectively on colloids. The detections of biocolloids by LIBD-based NPA as described in this paper for the first time clearly reveal that the method is well suited for detecting not only non-biological particulate matter in water, but also microorganisms in the transition range between solid and liquid matter. Consequently, the method can be used for online-monitoring, for example of both the non-biological and biological particulate content during the purification, transport and storage of drinking water. It is further possible to monitor online contamination of pure water or process chemicals by biological matter e.g. in biomedical industry.
\end{abstract}

Keywords: Biocolloid, microorganism, plasma detection, LIBD, Nano-Particle Analyzer

\section{Introduction}

Natural colloids are omnipresent and may differ considerably in terms of the parameters of size, density, chemical composition, shape, and surface charge [13]. Colloids are understood to be particles, the diameter of which may range from about $1 \mathrm{~nm}$ to $1 \mu \mathrm{m}[2,10,21]$. Due to their very small size, nanoparticles/colloids possess a high surface-to-mass ratio. It is responsible for the high sorption capacities towards numerous substances, as a result of which colloids are able to stabilize substances/pollutants in a solution even beyond the thermodynamic solubility of the respective compound $[15,19]$.

Colloids are also important to the mass balance of lakes and oceans, as a number of nutrients, such as phosphorus, nitrogen or elements relevant to phytoplankton metabolism, e.g. iron, may be bound colloidally. It was found that the predominant fraction of $\mathrm{Fe}$ in the North Atlantic and North Pacific is bound to colloids [27].

Moreover, colloids may be of biological nature (e.g. bacteria, spores, viruses) - they are frequently referred to as biocolloids - and, hence, have a decisive influence on the quality of e.g. drinking water. In addition, organic colloids represent a large proportion of nutrients for microorganisms in aquatic systems [20].

For the classification of aquatic particles their size ranges as well as some selected particle detection methods are illustrated in Fig. 1 [24].

\footnotetext{
*Corresponding author. Tel.: +49 724782 2648; Fax: +49 724782 6639; E-mail: tobias.bundschuh@web.de.
} 


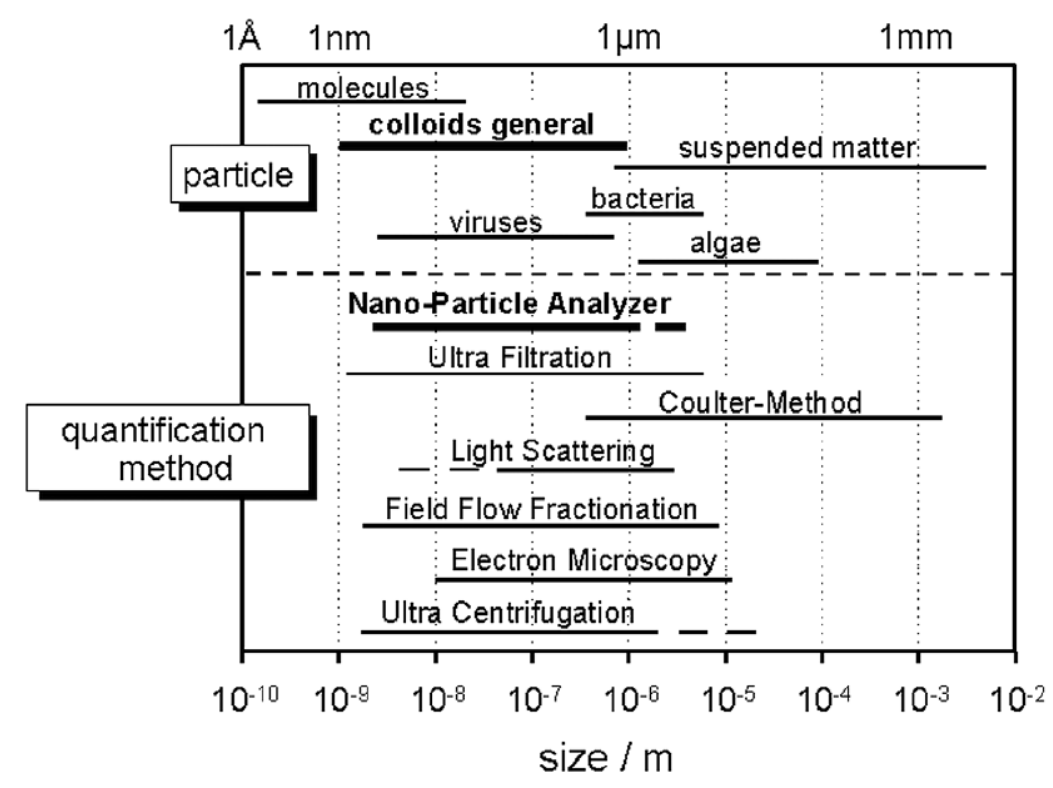

Fig. 1. Size spectrum of natural colloids and selected particle detection methods.

As far as the monitoring of drinking water during treatment, transportation, and storage as well as the protection of natural water resources are concerned, strict regulations exist or will be adopted in the near future, e.g. within the framework of the Water Safety Plans of the WHO (World Health Organization). Consequently, an efficient separation of particulate water contents during water treatment is of crucial importance and will even gain significance. To monitor this process and to check the water for its particulate concentration upon treatment and during storage and transportation in the distribution network of the water suppliers, highly sensitive particle detection methods are required also in the colloidal size range.

Furthermore, it is important to prevent contamination of raw products (e.g. pure water or process chemicals) and to maintain quality control of the finished products in pharmaceutical and biomedical industry. In many cases the regulations are becoming stricter, and it is of advantage to have one analysis method capable of monitoring online both non-biological and biological particulate material at the same time and in trace concentrations.

Quantification of aquatic colloids is associated with considerable difficulties, as these frequently exist in small concentrations only. Additionally, the majority of natural colloids has a diameter below $100 \mathrm{~nm}$ [16]. The analytical methods available for the characterization of nanoparticles, however, often possess too low a sensitivity in the particle range below $100-500 \mathrm{~nm}$ or require pre-concentration and separation processes that may lead to artifacts in highly sensitive colloid characterization. Therefore, non-invasive methods, such as laser light scattering methods or laser light obscuration are preferred. The measuring range of obscuration methods, however, does not extend down to the lower colloidal size range (detection limit: about 500-1000 nm). Light scattering methods frequently cannot be applied due to their very small sensitivity (see Fig. 5) [14,22]. In the past years, a novel colloid detection method with a sensitivity increased by several orders of magnitude was developed, namely, Laser-Induced Breakdown Detection (LIBD). Meanwhile, this method has been applied successfully for the high-sensitivity detection of particles in ultra-pure water used by semiconductor industry $[11,17]$ as well as for the characterization of various synthetic and natural aquatic colloids [3-5,18]. 


\section{LIBD-based nano-particle analyzer}

\subsection{Principle and setup of the nano-particle analyzer}

The LIBD-based Nano-Particle Analyzer (NPA/LIBD) generates a dielectric breakdown in the focal area of a pulsed laser beam. The power density required to generate a breakdown in a medium decreases from the gaseous to the solid state of aggregation of the medium [1]. Energy of the laser beam focused into the aqueous sample is set such that just no breakdown event is caused in ultra-pure water as dispersion agent, while plasma formation may take place on solid particles. Thus, plasmas are generated selectively on colloid particles; the associated light emissions are detected by means of a microscope/camera system $[7,12]$. Information on the particle size may be obtained from the spatial distribution of breakdown events in the focal area. This distribution is independent of particle concentration and increases with increasing particle diameter [5,7]. Particle concentration may be determined from the statistical frequency of events in combination with the previously obtained particle size [3,5-7,12,25].

The NPA/LIBD instrumentation is represented schematically in Fig. 2. A pulsed solid state laser (here: Nd:YAG, Continuum SureLite I) is applied for plasma generation. The laser is run at a repetition rate of $20 \mathrm{~Hz}$ and a wavelength of $532 \mathrm{~nm}$ (frequency-doubled basic wavelength). A small part of the laser beam is passed via a beam splitter to a calibrated energy detector (pyroelectric detector, Newport GmbH) that is connected with an energy control unit and a computer. This newly designed system of software and hardware components allows for a fully automatic operation also via a remote interface [26]. Among others, laser energy is adjusted and controlled by a central micro controller (nanomodule). The main part of the laser beam is focused into the sample cell ( $3.5 \mathrm{ml}$ quartz glass cuvette, Hellma $\mathrm{GmbH}$ ) containing the sample via a plano-convex lens (focal length $60 \mathrm{~mm}$, Linos $\mathrm{GmbH}$ ). The plasma light emissions generated are magnified by a microscope (Leica $\mathrm{GmbH}$ ), recorded by means of a camera (Basler $\mathrm{GmbH}$ ), and transmitted to a PC for data storage and evaluation.

\subsection{Calibration and parameterization of the nano-particle analyzer}

To calibrate the NPA/LIBD system, various diluted polystyrene-colloid standard dispersions (Polymer Standards Service $\mathrm{GmbH}$ ) with particle diameters of $19 \pm 1.5 \mathrm{~nm}$ to $1020 \pm 22 \mathrm{~nm}$ were used. In addition, also inorganic colloid standards were applied. The initial standard particle solutions were diluted to the concentration desired using ultra-pure water from a MilliQ synthesis A10 facility (Millipore $\mathrm{GmbH}$ ) or an ARIUM 611 UF facility (Sartorius AG). The samples were measured in quartz glass cuvettes with

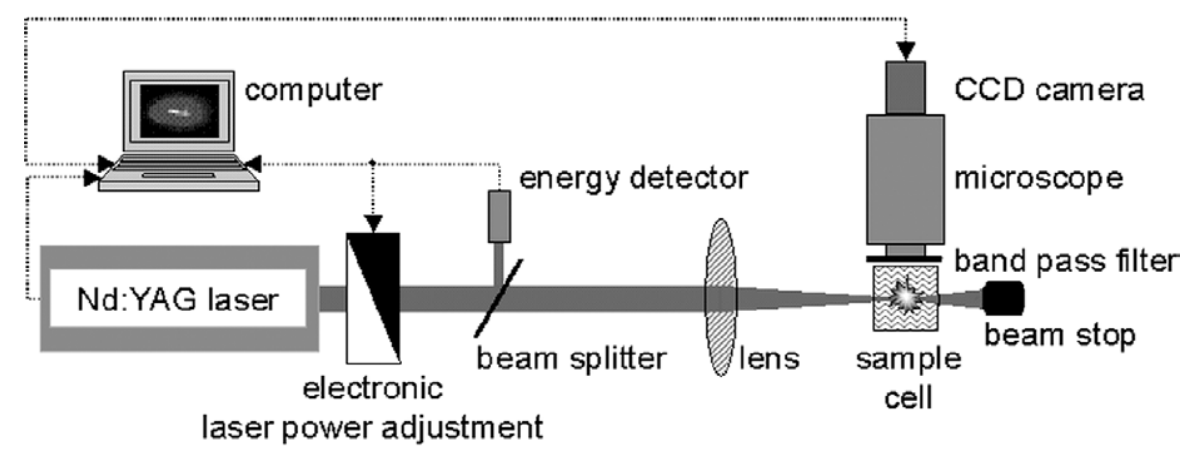

Fig. 2. Schematic representation of the NPA/LIBD instrumentation. 
an optical length of $10 \mathrm{~mm}$ and an optical width of $10 \mathrm{~mm}$ (volume $3.5 \mathrm{ml}$ ). All experiments were performed at normal atmospheric pressure and room temperature.

To ensure a plasma ignition selectively on colloids, it is made use of the fact that the power density required to form a plasma is smaller for solids than for liquids. For this reason, laser power density of the NPA/LIBD is reduced such that no plasma is generated in the dispersion agent (ultra-pure water). Still, power density has to be sufficient to generate breakdown events on colloids. Under constant optical and experimental conditions (focal length of lens, wavelength of laser light, laser pulse duration, beam diameter), laser pulse energy may be considered instead of laser pulse power density. For this purpose, the breakdown probabilities (number of plasma events per number of laser pulses) are recorded as a function of the incident laser pulse energy for both ultra-pure water and a dispersion with spherical $22 \mathrm{~nm}$ polystyrene colloids of $10 \mathrm{ppt}$ (app. $10 \mathrm{ng} / \mathrm{l}$ ) in concentration. Then, a comparison is made. Determination of the breakdown probability at variable laser pulse energies yields the sigmoidal curves described in literature $[3,6,18]$. From these, the laser pulse energy is obtained, at which just no breakdown is caused in ultra-pure water. In the system used here, a laser energy of $0.52 \pm 0.02 \mathrm{~mJ}$ results in a breakdown probability of $0.001 \pm 0.001$ in ultra-pure water. In contrast to this, significantly increased breakdown probabilities of $0.18 \pm 0.02$ (factor of 180) are recorded in the polystyrene dispersion (particle diameter: $22 \mathrm{~nm}$; particle concentration: $10 \mathrm{ppt}$ ).

To determine the breakdown probability as a function of colloid concentration, dispersions of the individual colloid standards with concentrations ranging from a few ppt up to several ppm are measured. Using a theoretical model [23] based on binomial statistics, the NPA/LIBD instrumentation is then parameterized according to the procedure described in $[3,6,8,18,23]$.

To determine number-weighted, mean particle sizes of colloids in unknown samples, it is made use of the fact that the probability of igniting a plasma on a particle depends on the power density of the laser pulse at the particle location and the number of weakest bound electrons of the particle. This means that an increasing number of these so-called initial electrons are available with increasing particle size. With increasing particle size, a breakdown may therefore be generated at decreasing laser pulse power densities. For this reason, particles with increasing size are increasingly able to ignite in the energy-depleted areas of the focus. By recording a sufficient number of plasmas, a spatial distribution of breakdown events is obtained, which reflects the ignition range of colloids in the focus (so-called ignition length $L_{Z}(P)$ ). This means that with increasing particle size of the colloids contained in the dispersion, the ignition range in the focus (plasma event volume) and, hence, the effective focal length (ignition length) are increased $[7,12]$. To determine the two-dimensional projection of the plasma event volume of standard colloids, each size of the colloid standards is measured until several thousands of plasma events have been detected. By plotting the obtained ignition length of every colloid size versus the corresponding particle diameter and fitting the data points using a fit algorithm, a mean particle size (colloid standard-equivalent) results for colloids in unknown samples [3,7,12]. In combination with concentration calibration and parameterization, particle concentration can be calculated using the procedure described in $[3,5,9]$.

\section{Preparation of biocolloid suspensions and corresponding blanks}

It was decided, to use two different suspensions as model biocolloids: on one hand spores of Bacillus subtilis, on the other hand the bacteria Enterococcus faecalis. 
From Bacillus subtilis-spores (Merck GmbH, No. 1.10649) five ampulles were filtered on a $0.2 \mu \mathrm{m}$ polycarbonate filter (Millipore GmbH, No. GTTP04700) and washed thereon with 2-times $150 \mathrm{ml}$ ultrapure water. The filter was put in $40 \mathrm{ml}$ ultra-pure water and shaken $10 \mathrm{~min}$ to remove the spores from the filter. This spore solution was examined by NPA.

An Enterococcus faecalis suspension was produced by inoculation of the culture (DSMZ 20478) on Agar 220 (DSMZ) for $24 \mathrm{~h}$ at $36^{\circ} \mathrm{C}$. After incubation $10 \mathrm{ml}$ ultra-pure water were added and the bacteria were removed from the agar with the help of a sterile inoculating loop. After addition of further $4 \mathrm{ml}$ ultra-pure water and vigorous mixing the water with the bacteria was filtered on a sterile $0.2 \mu \mathrm{m}$ polycarbonate filter (see above). They were washed with 2-times $150 \mathrm{ml}$ ultra-pure water on the filter. The filter was put in $40 \mathrm{ml}$ ultra-pure water and shaken $10 \mathrm{~min}$ to remove the bacteria from the filter. This bacteria solution was examined by NPA.

As blanks the same treatments of ultra-pure water filtered over polycarbonate filter, filter washing and filter shaking was used, but without previous adding bacteria or spores on the filter.

The concentration of the bacteria and spore suspension, respectively, was quantified in both cases by colony counts on plate count agar (DEV) at $22^{\circ} \mathrm{C}$ for 7 days. The concentration of the spore suspension was $3.2 \times 10^{6} \mathrm{cfu} / \mathrm{ml}\left(\mathrm{cfu}=\right.$ colony forming unit), of the bacteria suspension $4.0 \times 10^{7} \mathrm{cfu} / \mathrm{ml}$.

\section{Biocolloid detection by NPA}

The power density necessary for the generation of a laser-induced breakdown is dependent on the state of aggregation of the respective medium. To verify that the NPA/LIBD is also capable of detecting structures in the transition range between solid and liquid matter, i.e. which contain a high amount of liquid, suspensions of microorganisms as described in Section 3 were used; the main characteristics of the microorganisms are shown in Table 1 . Unlike the spores which are a rather "compact" permanent form of bacteria with a comparatively low water content (typically 13-15\%), the bacteria themselves contain around $70-85 \%$ water. Both types have been examined with the NPA instrumentation; for this purpose the breakdown probabilities of both the dispersions of microorganisms and their corresponding blanks were recorded as a function of the incident laser energy.

In Fig. 3, the breakdown probabilities are plotted versus an increasing laser pulse energy for a suspension with spores of Bacillus subtilis in comparison to the respective blank (see Section 3). Compared to the breakdown probability obtained for spores of the type Bacillus subtilis, however, the blank curve is located at significantly increased laser pulse energies. At a pulse energy of $0.24 \mathrm{~mJ}$ (see vertical dashed

Table 1

Main characteristics of the examined biocolloids

\begin{tabular}{lcccc}
\hline Type & Form & $\begin{array}{c}\text { Water content } \\
(\%)\end{array}$ & $\begin{array}{c}\text { Size } \\
(\mu \mathrm{m})\end{array}$ & $\begin{array}{c}\text { Concentration } \\
(\mathrm{CFU} / \mathrm{ml})^{*}\end{array}$ \\
\hline $\begin{array}{l}\text { Spores } \\
\text { Bacillus subtilis }\end{array}$ & elliptical & $13-15$ & $\approx 0.8 \times 1.5-1.8$ & $3.2 \times 10^{6}$ \\
$\begin{array}{l}\text { Bacteria } \\
\text { Enterococcus faecalis }\end{array}$ & spherical & $70-85$ & $\approx 1$ & $4.0 \times 10^{7}$ \\
$\begin{array}{l}\text { Bacteria } \\
\text { Enterococcus durans }\end{array}$ & spherical & $70-85$ & $\approx 1-2$ & $2.0 \times 10^{7}$ \\
\hline
\end{tabular}

${ }^{*} \mathrm{CFU}=$ colony forming units (determination of colonies on plate count agar (DEV): $22^{\circ} \mathrm{C}, 7$ days).

${ }^{* *}$ Wagner et al., Acta Hydrochim. Hydrobiol. 30 (2002), 266-274. 


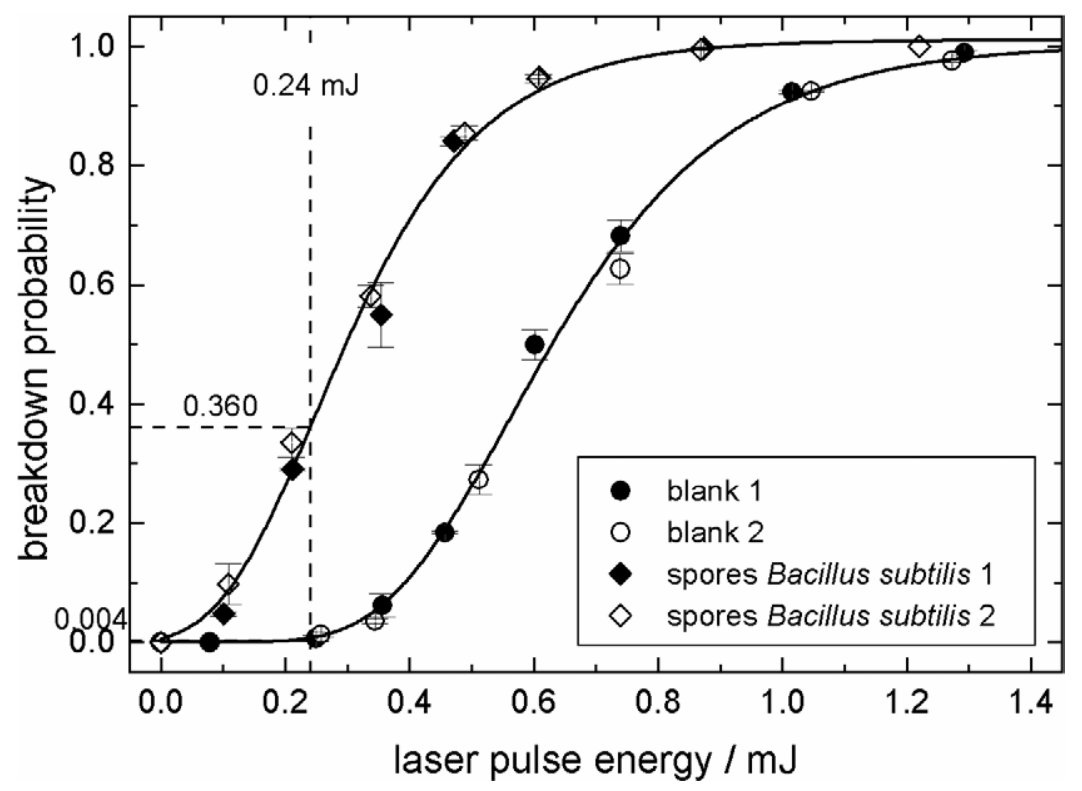

Fig. 3. Breakdown probability as a function of laser pulse energy. Compared to the blank, the spore suspension of Bacillus subtilis exhibits a significantly increased number of plasma events (see horizontal dashed lines at $0.24 \mathrm{~mJ}$ ).

line in Fig. 3), for instance, the blank exhibits a breakdown probability of 0.004 , whereas the spore suspension reaches a value of 0.360 , i.e. a plasma formation rate increased by a factor of 90 .

In another experiment with living bacteria of the type Enterococcus faecalis the bacteria suspension and the corresponding blank were produced in the way described above. Figure 4 presents the breakdown probabilities for the bacteria suspension of Enterococcus faecalis, both undiluted and diluted with ultrapure water in the ratio of $1: 1$, as well as for the corresponding blank solution. The difference between the blank and the bacteria suspension is not as large as for the spore suspension. However, the value of the bacteria suspension of the type Enterococcus faecalis is significant and exceeds the blank value by a factor of app. 10 (at a laser pulse energy of $0.24 \mathrm{~mJ}$ (see vertical dashed line in Fig. 4), the blank exhibits a breakdown probability of 0.004 , whereas the value of the bacteria suspension amounts to 0.041 ). The sigmoidal curve only becomes flatter and approaches the curve of the blank. This is clearly reflected by the concentration of the bacteria suspension being about half (open diamonds in Fig. 4) that of the undiluted suspension (solid diamonds in Fig. 4). It is clearly visible that the laser pulse energy, at which the curve significantly exceeds the background value (breakdown threshold), does not depend on the concentration, but on the size and the type of material of the particles in the sample.

From a previous similar experiment on bacteria of the type Enterococcus durans, again, plotting of breakdown probabilities as a function of laser pulse energy yields the typical sigmoidal curves. In this case, too, the difference between the blank and the bacteria suspension was significantly higher by a factor of 16 (at a laser pulse energy of $0.4 \mathrm{~mJ}$ the breakdown probability of the bacteria suspension was 0.065 , whereas that of the blank was only 0.004); the difference was larger compared to Enterococcus faecalis (factor of 10). E. durans bacteria have a size of about 1-2 $\mu \mathrm{m}$ and, hence, are larger than the E. faecalis bacteria of about $1 \mu \mathrm{m}$. Consequently, they can be detected by the NPA/LIBD at a smaller laser pulse energy. Here, it is assumed that both bacteria types have a similar material breakdown threshold. As a result, a larger difference of the breakdown probabilities is observed at a constant pulse energy in comparison to the blank. 


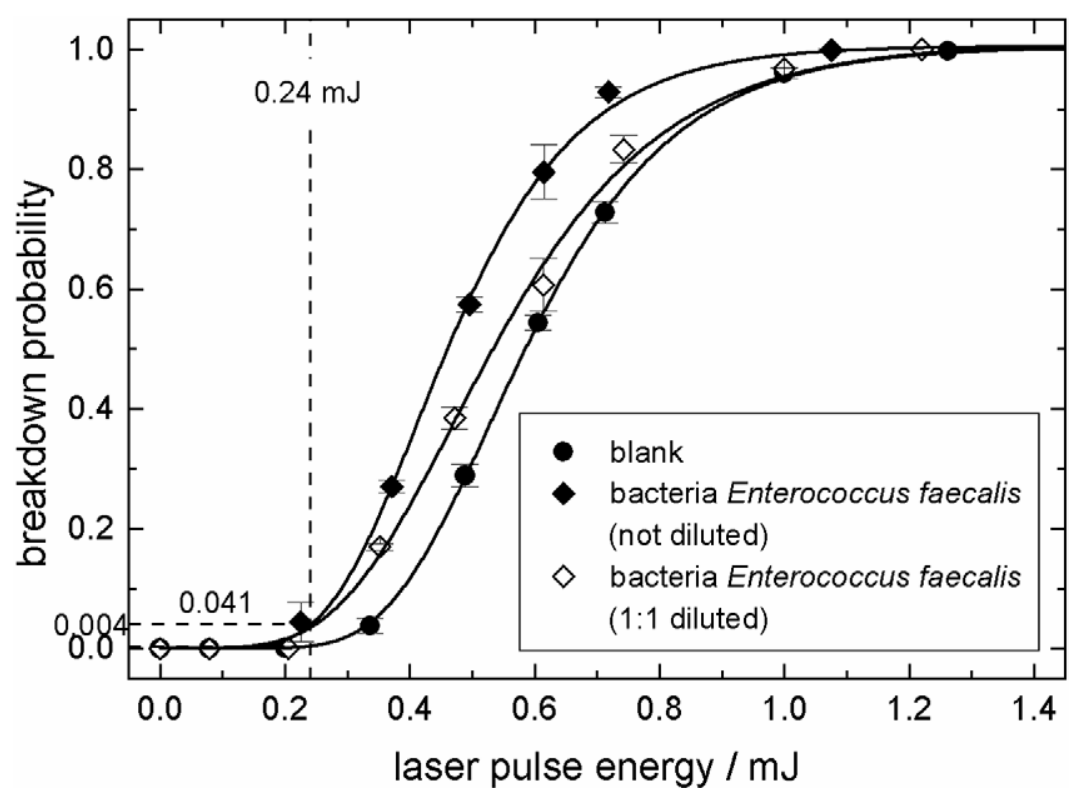

Fig. 4. Breakdown probability as a function of laser pulse energy. Compared to the blank, the bacteria suspension of Enterococcus faecalis shows a significantly increased number of plasma events (see horizontal dashed lines at $0.24 \mathrm{~mJ}$ ).

\section{Conclusions}

The novel conception of the Nano-Particle Analyzer on the basis of Laser-Induced Breakdown Detection (NPA/LIBD) allows for a fully automatic operation with the exception of sample exchange. All major functions of the NPA may be operated via a remote interface. Fully automatic operation of the NPA requires communication between the software and the individual hardware components of the instrumentation. This is achieved by a central nanomodule (micro controller) that is connected to the control PC via a serial interface RS232. Via a special protocol, it accepts the necessary orders and supplies the required information. A major task of this nanomodule control unit consists in the adjustment and control of laser pulse energy. The energy signal coming from the calibrated pyroelectric energy detector (see Fig. 2) is digitized, compared with the value given by the software, and, if necessary, corrected by shifting the attenuator. In addition, the hardware unit controls the mechanical shutter that can shut off the laser beam before it is focused by the lens. A cuvette sensor serves to inform the system as to whether a sample cell is present and has been inserted correctly. Connection of an autosampler has been prepared.

To calibrate the NPA, various organic, inorganic, and biological standards (if available) may be applied. It is possible to retrieve several calibration profiles in parallel and to use them for a measurement, depending on the sample investigated. In this way, chemism of the particles (if known) can be considered in the evaluation. Particle shape may also be included in the evaluation; several shapes may be selected. If applicable, any shape that can be described mathematically can be taken up in the list. By recording several thousands of plasma events, the ignition length $L_{Z}(P)$ (see annotation in Section 2.2), and, hence, the number-weighted mean size of the particles investigated is determined. Together with the breakdown probability obtained, the system automatically calculates a colloid number concentration with all device-specific parameters, e.g. laser wavelength $\lambda$, beam waistline $\omega_{F}$, minimum particle radius $R_{\min }$, and refraction index $n(\lambda)$ of the dispersion medium, being taken into account. With the 


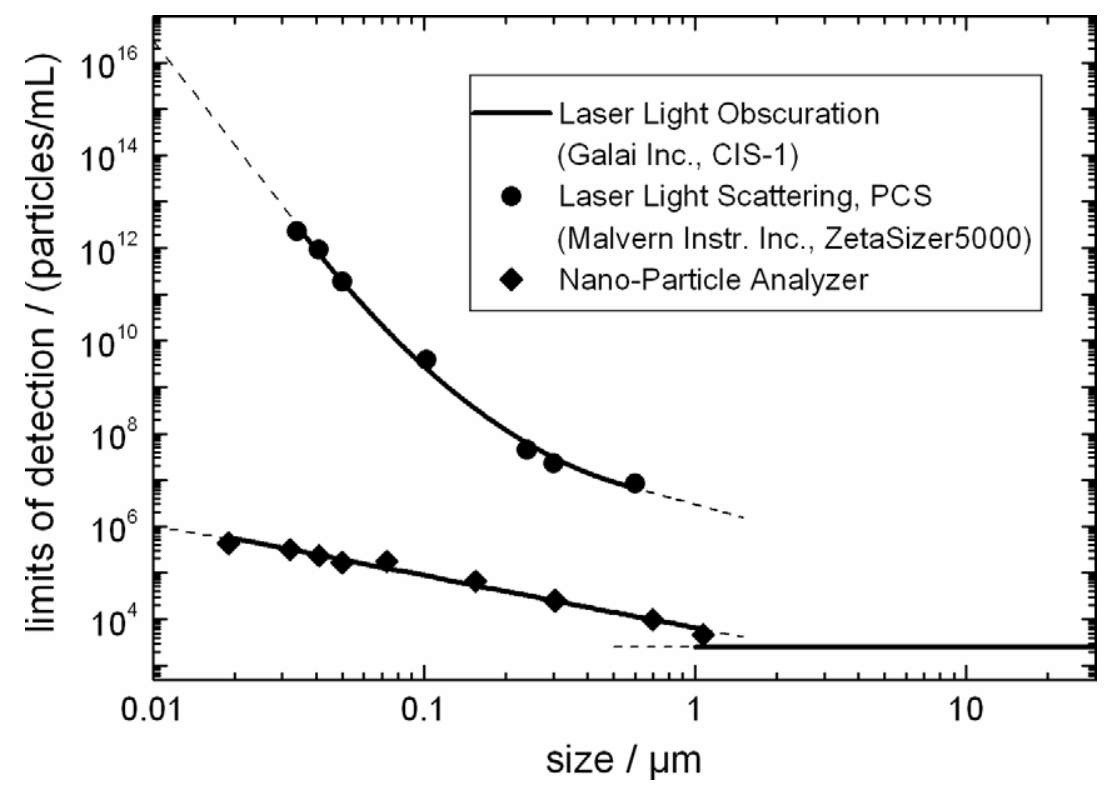

Fig. 5. Comparison of detection limits of the non-invasive particle detection methods PCS (photon correlation spectroscopy, Malvern Instruments Inc., Zetasizer 5000), laser light obscuration (Galai Inc., CIS-1), and NPA/LIBD (Nano-Particle Analyzer based on LIBD) (modified according to [14]).

particle shape and the densities of the dispersion medium and the particles being considered, a colloid mass concentration can be calculated.

The experimental measurements are "absolute", which means that sigmoidal curves of both test campaigns (this work and [25]) cannot be compared directly, as the NPA instrumentation and, hence, the optical parameters were changed between the campaigns. On the other hand, when determining the size and concentration of particles in an unknown sample, the values can be compared directly, because in this cases the possibly varied optical parameters between the measurements or varying facilities are always considered in the calculations.

The major advantage of the NPA as compared to conventional methods like laser light scattering is a sensitivity increase by several orders of magnitude (for app. $20 \mathrm{~nm}$ particles, the detection limit is about 1,000,000 times better than for PCS). For comparison, the detection limits of PCS, laser light obscuration, and NPA are illustrated in Fig. 5.

Microorganisms may contribute to the particulate pollution of natural water bodies. As the nanoparticle analyzer is based on the varying plasma ignition behavior of solid and liquid matter, structures like bacteria and micelles, whose main constituent is water, are more difficult to detect in principle. Using various, well cultivable microorganisms of about 0.8 to about $2 \mu \mathrm{m}$ in size, it was demonstrated that the NPA/LIBD method is suited in principle for the detection of such structures. This is reflected by the comparison of breakdown probabilities obtained as a function of laser pulse energy for the microorganism suspensions, and the corresponding blanks. Here, the suspensions revealed a much smaller breakdown threshold than the blanks, i.e. plasma events were observed at significantly smaller laser pulse energies. Thus, it was shown for the first time clearly that also colloids in the transition range between solid and liquid matter can be detected by the NPA/LIBD. It must be emphasized that the NPA/LIBD yields a number-weighed mean particle diameter and, hence, a mean particle concentration that is made up of all particles existing in the sample to be analyzed. It cannot be distinguished between various particles; the 
measurement value obtained in the current development stage is a sum parameter of both biological and non-biological colloids. In the future, however, the NPA might be applied as a simply designed, small, low-cost device, in which a warning will be issued when a particle concentration threshold defined according to the application is exceeded; this would then initiate further analysis (monitoring function). Water suppliers, for example, might install such NPA/LIBD-based detectors downstream of the treatment stage and at exposed positions of their partly very long water distribution networks (e.g. $1700 \mathrm{~km}$ at the Zweckverband Bodensee-Wasserversorgung) for online-control of the water quality parameter.

In pharmaceutical and biomedical industry the NPA could be used to monitor pure process waters and chemicals; any particulate contamination can be detected in an online manner and in trace concentrations. The design of the instrumentation makes it even possible to monitor the liquids within closed pipe systems since the laser beam can be focused directly into the pipe through a glass window or a transparent pipe section. In this context it is interesting to know that latest experiments showed that the NPA is also capable of detecting viruses; work in this field is still in progress.

\section{Acknowledgement}

We would like to thank Mrs. Dipl.-Übers. Maike Schröder from the Translation Services Division of Forschungszentrum Karlsruhe $\mathrm{GmbH}$ for the translation of this manuscript into English.

\section{References}

[1] J.R. Bettis, Correlation among the laser-induced breakdown thresholds in solids, liquids, and gases, Appl. Opt. 31 (1992), $3448-3452$.

[2] J. Buffle and G.G. Leppard, Characterization of aquatic colloids and macromolecules. 1. Structure and behavior of colloidal material, Environ. Sci. Tech. 29 (1995), 2169-2175.

[3] T. Bundschuh, Entwicklung und Anwendung der Laser-induzierten Breakdown-Detektion zur Quantifizierung aquatischer Kolloide und Actinidenkolloide, Ph.D. Dissertation, Technical University Munich, Germany, 1999.

[4] T. Bundschuh, R. Knopp, R. Müller, J.I. Kim, V. Neck and T. Fanghänel, Application of LIBD to the determination of the solubility product of thorium(IV)-colloids, Radiochim. Acta 88 (2000), 625-632.

[5] T. Bundschuh, R. Knopp, R. Winzenbacher, J.I. Kim and R. Köster, Quantification of aquatic nano particles after different steps of Bodensee water purification with Laser-Induced Breakdown Detection (LIBD), Acta Hydrochim. Hydrobiol. 29 (2001), 7-15.

[6] T. Bundschuh, R. Knopp and J.I. Kim, Laser-Induced Breakdown Detection (LIBD) of aquatic colloids with different laser systems, Colloids Surf. A 177 (2001), 47-55.

[7] T. Bundschuh, W. Hauser, J.I. Kim, R. Knopp and F.J. Scherbaum, Determination of colloid size by 2-D optical detection of laser induced plasma, Colloids Surf. A 180 (2001), 285-293.

[8] T. Bundschuh, T. Wagner and R. Köster, Laser-Induced Breakdown Detection (LIBD) for highly sensitive quantification of aquatic colloids. Part I: Principle of LIBD and mathematical model, Particle and Particle Systems Characterization, submitted.

[9] T. Bundschuh, T. Wagner and R. Köster, Hochsensitive Partikelbestimmung mittels der Laser-induzierten Breakdown Detektion, Chem. Ing. Tech. 75 (2003), 386-390.

[10] D.H. Everett, Basic Principles of Colloid Science, Royal Society of Chemistry, London, UK, 1989.

[11] H. Fujimori, T. Matsui, T. Ajiro, K. Yokose, Y. Hsueh and S. Izumi, Detection of fine particles in liquids by laser breakdown method, Jpn. J. Appl. Phys. 31 (1992), 1514-1518.

[12] W. Hauser and T. Bundschuh, Verfahren zur Bestimmung der Größe von Partikeln in einer Lösung. Patent DE 19833339 C1 (Internat. Public. No.: WO 00/06993), Munich, Germany, 2000.

[13] T. Hofmann, T. Baumann, T. Bundschuh, F. von der Kammer, A. Leis, D. Schmitt, T. Schäfer, J. Thieme, K.U. Totsche and H. Zänker, Aquatische Kolloide I: Eine Übersichtsarbeit zur Definition, zu Systemen und zur Relevanz, Grundwasser 8 (2003), 203-212. 
[14] T. Hofmann, T. Baumann, T. Bundschuh, F. von der Kammer, A. Leis, D. Schmitt, T. Schäfer, J. Thieme, K.U. Totsche and H. Zänker, Aquatische Kolloide II: Eine Übersichtsarbeit zur Probenahme, Probenaufbereitung und Charakterisierung, Grundwasser 8 (2003), 213-223.

[15] B.D. Honeyman and P.H. Santschi, The role of particles and colloids in the transport of radionuclides and trace metals in the oceans, in: Environmental Particles, J. Buffle and H.P. van Leeuwen, eds, Vol. 1, 1st edn, Lewis Publishers Inc., Chelsea, 1992, pp. 379-423.

[16] J.I. Kim, Actinide colloids in natural aquifer systems, MRS Bulletin XIX (1994), 47-52.

[17] T. Kitamori, K. Yokose, M. Sakagami and T. Sawada, Detection and counting of ultrafine particles in ultra-pure water using laser breakdown acoustic method, Jpn. J. Appl. Phys. 28 (1989), 1195-1198.

[18] R. Knopp, Laserinduzierte Breakdowndetektion zur Charakterisierung und Quantifizierung aquatischer Kolloide, Ph.D. Dissertation, Technical University Munich, Germany, 1996.

[19] J.F. McCarthy and J.M. Zachara, Subsurface transport of contaminants, Environ. Sci. Technol. 23 (1989), 496-502.

[20] U. Münster, Concentration and fluxes of organic carbon substrates in the aquatic environment, Antonie von Leeuwenhoek 63 (1993), 243-274.

[21] D. Myers, Surfaces, Interfaces and Colloids, VCH Publishers Inc., New York, 1991.

[22] M. Plaschke, T. Schäfer, T. Bundschuh, T. Ngo Manh, R. Knopp, H. Geckeis and J.I. Kim, Size characterization of bentonite colloids by different methods, Anal. Chem. 73 (2001), 4338-4347.

[23] F.J. Scherbaum, R. Knopp and J.I. Kim, Counting of particles in aqueous solutions by laser induced breakdown photoacoustic detection, Appl. Physics B 63 (1996), 299-306.

[24] W. Stumm, Chemical interaction in practical separation, Env. Sci. Technol. 11 (1977), 1066-1069.

[25] T. Wagner, T. Bundschuh, R. Schick, T. Schwartz and R. Köster, Investigation of colloidal water content with laser-induced breakdown detection during drinking water purification, Acta Hydrochim. Hydrobiol. 30 (2002), 266-274.

[26] T. Wagner, T. Bundschuh and R. Köster, Laser-Induced Breakdown Detection (LIBD) for highly sensitive quantification of aquatic colloids. Part II: Experimental setup of LIBD and applications, Particle and Particle Systems Characterization, submitted.

[27] J. Wu, E. Boyle, W. Sunda and L.-S. Wen, Soluble and colloidal iron in the oligotrophic north Atlantic and north Pacific, Science 293 (2001), 847-849. 


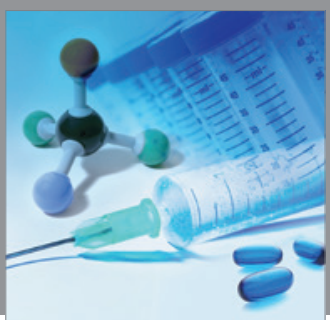

International Journal of

Medicinal Chemistry

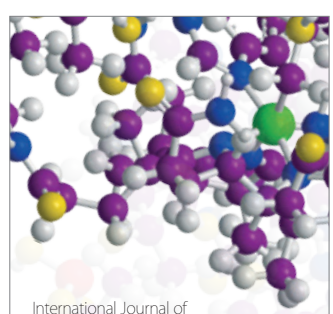

Carbohydrate Chemistry

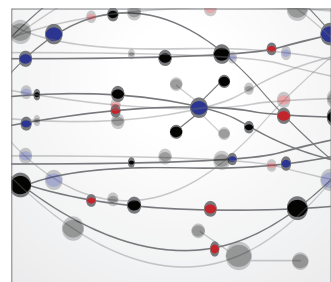

The Scientific World Journal
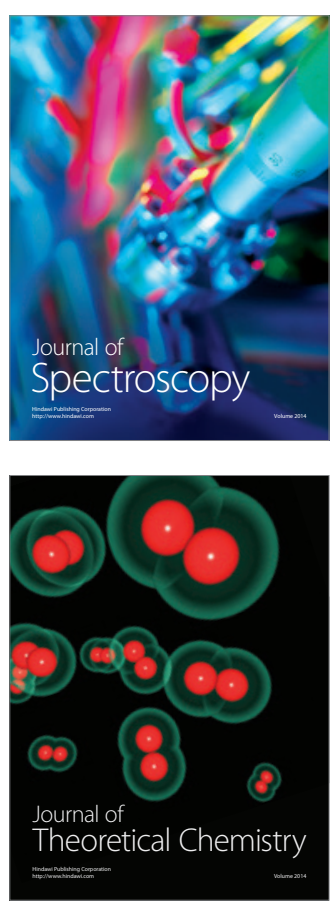
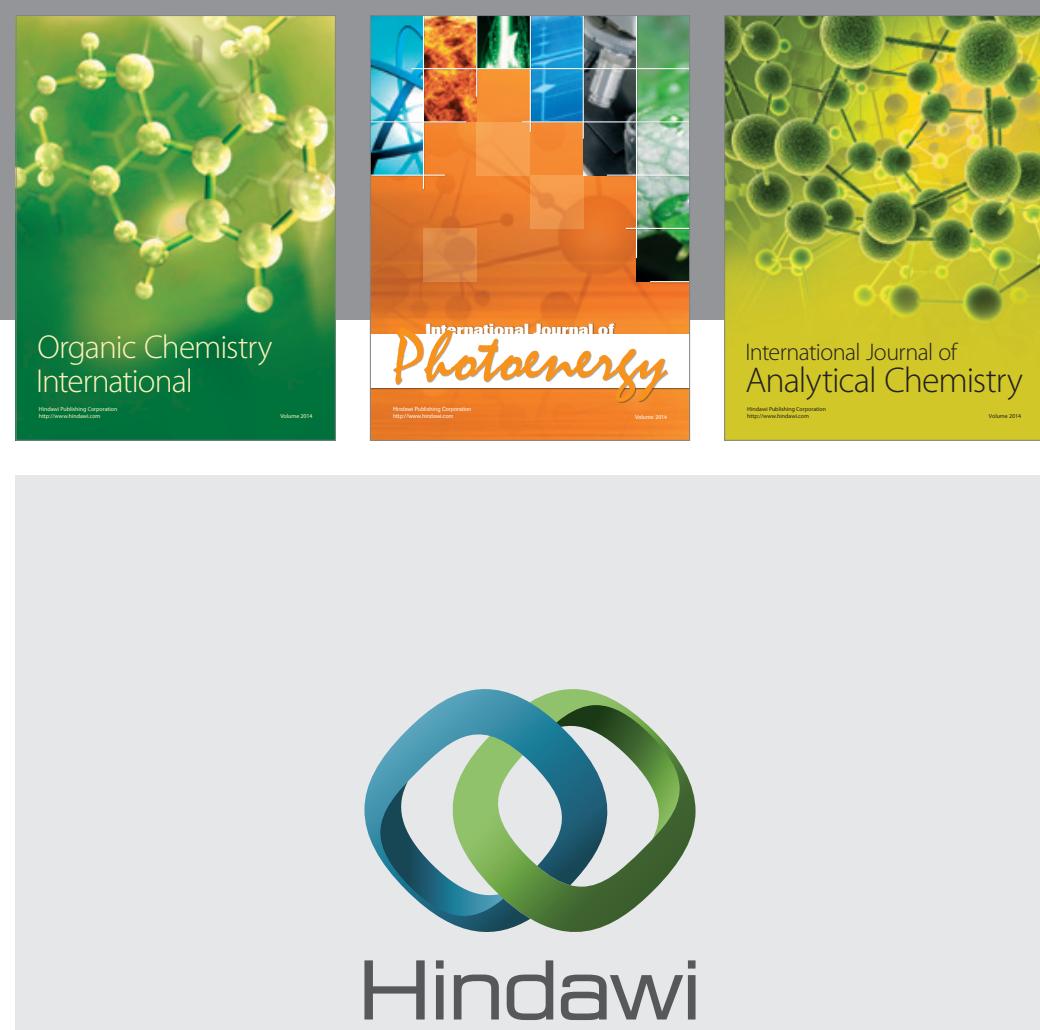

Submit your manuscripts at

http://www.hindawi.com
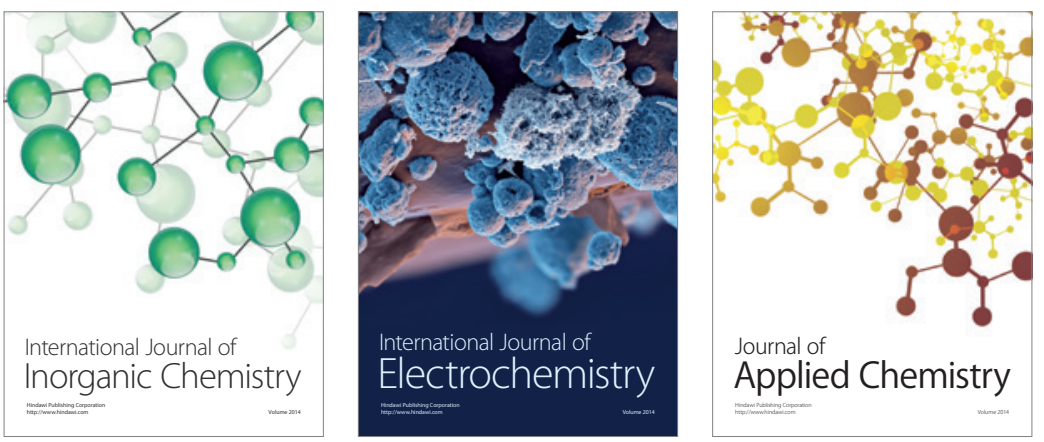

Journal of

Applied Chemistry
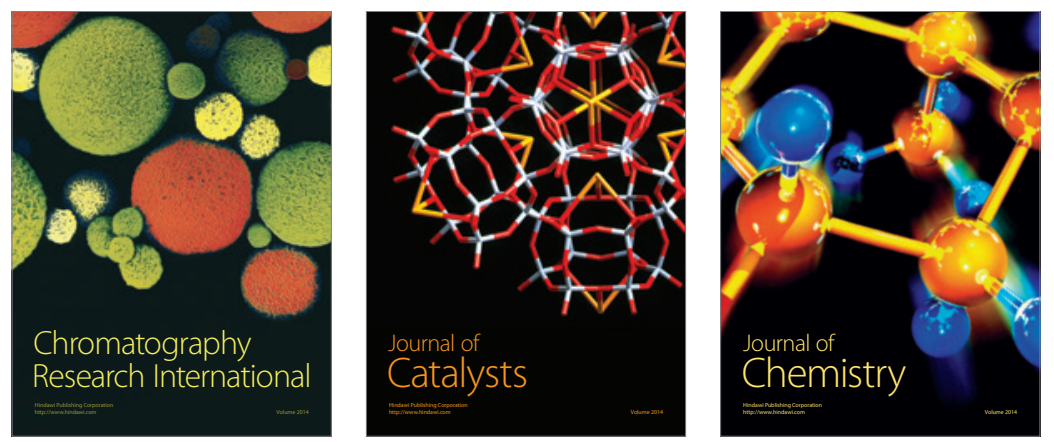
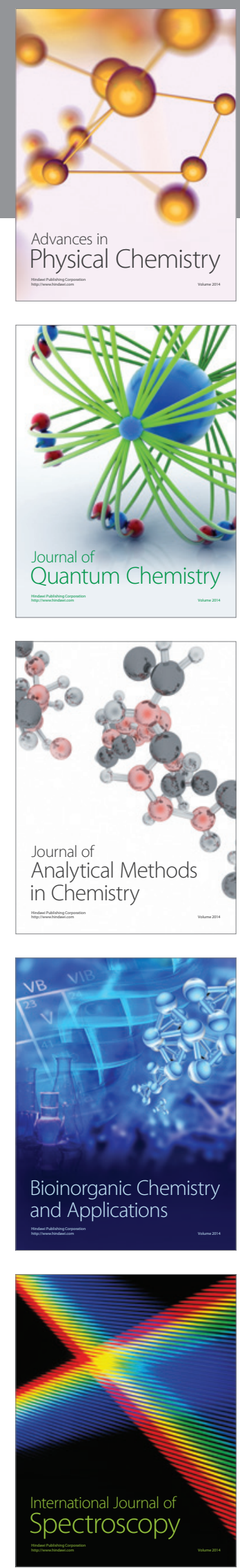